\title{
Bioavailability assessment of topical delivery systems: Effect of vehicle evaporation upon in vitro delivery of minoxidil from solution formulations
}

\author{
Chia-Ming Chiang ${ }^{1}$, G.L. Flynn ${ }^{1}$, N.D. Weiner ${ }^{1}$ and G.J. Szpunar ${ }^{2}$ \\ ${ }^{\prime}$ College of Pharmacy, University of Michigan, Ann Arbor, MI 48109 (U.S.A.) and ${ }^{2}$ The Upjohn Company, Kalamazoo, MI (U.S.A.)
}

(Received 14 October 1988)

(Modified version received 12 January 1989)

(Accepted 28 April 1989)

Key words: Topical; Vehicle; Thin application; Crystallization; Permeability; Flux

\section{Summary}

Two sets of in vitro permeation experiments were carried out to study the topical delivery of minoxidil as a function of its thermodynamic activity in the hydroalcoholic vehicles applied to the skin. Franz diffusion cells were employed with human cadaver skin membranes. The delivery of minoxidil of different concentrations $(0.5 \%, 1 \%, 2 \%, 3 \%, 4 \%$ and $5 \%)$ from simple solutions in a fixed composition of propylene glycol/water/ethanol $(20.0: 63.2: 16.8)$ was determined. The flux of minoxidil increased systematically from $0.5 \%$ to $3 \%$ concentration and then fell back abruptly at the $5 \%$ concentration. The maximum in the flux seen at the intermediate concentrations (3-4\%) opened the possibility that the drug might be crystallizing upon evaporation of the volatile components of the vehicle. It was discovered that the $3 \%$ and even the $4 \%$ minoxidil concentration supported a relatively persistent supersaturation but at $5 \%$, the drug crystallized soon after its application. These phenomena were confirmed by observing crystal formation of minoxidil solutions under the microscope. It was found by following the weight of minoxidil solutions applied to a glass surface that $65 \%$ of the weight of the solution vehicle evaporated after the first 30 min and about $75 \%$ evaporated after 2 h. For the $5 \%$ minoxidil solution, crystals were evident within 6-7 min. By way of contrast, no crystals were evident in the $3 \%$ minoxidil solution after $2 \mathrm{~h}$. Secondly, since the evaporation of the volatile ethanol and water components of the vehicle play an important role in delivery, permeation profiles of minoxidil $(1 \%, 2 \%, 3 \%$ and $5 \%$ ) from solutions of propylene glycol, water and ethanol prepared so as to contain a fixed amount of propylene glycol per unit weight of minoxidil were obtained. There was no significant difference in the flux of minoxidil when its concentration in the non-volatile component, propylene glycol, was kept constant.

\section{Introduction}

The efficacy of topical formulations is dependent on the ability of the drugs they contain to be released from their vehicle and diffuse onto and through the skin, especially the skin's outermost

Correspondence: N.D. Weiner, College of Pharmacy, University of Michigan, Ann Arbor, MI 48109, U.S.A. layer, the stratum corneum. As a passive diffusion process, the driving force for diffusion is the gradient of the chemical potential and therefore, the flux of a given drug through the skin is a function of the thermodynamic activity of the drug in the vehicle. For vehicles containing volatile components, the flux of a given drug will change as the vehicle evaporates and is expected to be proportional to both the concentration of the drug in the vehicle and its activity until satura- 
tion is achieved, which normally sets the maximum thermodynamic activity. At this time, the drug is anticipated to precipitate, whereupon its flux should remain constant as set by the driving force of the saturated solution. However, the permeation of drug from complex vehicles is a dynamic process involving many changes and the thermodynamic and kinetic determinants of delivery are far more complex than established by this simple concept. Consequently, as the properties of a vehicle change continually as the volatile ingredients in the vehicle evaporate, the drug may supersaturate and flux levels above those set by a saturated solution may be obtained.

The purpose of this paper is to study the kinetics of topical delivery of drugs as a function of their thermodynamic activities in their vehicles. The prototype drug used for these purposes was minoxidil, a topical agent shown to be of some benefit in the treatment of alopecia areata (Weiss et al., 1981). The delivery of minoxidil at various concentrations from solutions composed of propylene glycol, water and ethanol was determined. The evaporation of ethanol and water causes relatively rapid compositional changes and hence the thermodynamic activity of the drug in the model vehicle is forced through dramatic changes. How the momentary flux of minoxidil relates to these changes tells much about the mechanisms of drug delivery from topical films.

\section{Materials and Methods}

\section{Materials}

Minoxidil, USP milled, was supplied by the Upjohn Co. (Kalamazoo, MI). The Ipyrimidine-2-

${ }^{14} \mathrm{C}$ mminoxidil was similarly obtained. It had a stated radiochemical purity of $99 \%$ and a sp. act. of $15.79 \mathrm{mCl} / \mathrm{mmol}$. Distilled water was used in the formulations. All other chemicals used were reagent grade.

\section{Preparation of minoxidil formulations}

A molecular mixture of radiolabelled and cold minoxidil was prepared by the following procedure. About $10 \mathrm{mg}$ of $\left[{ }^{14} \mathrm{C}\right]$ minoxidil crystals were added to about $200 \mathrm{mg}$ of unlabeled minoxidil in a $200 \mathrm{ml}$ flask and $6 \mathrm{ml}$ warm ethanol solution were added to dissolve the crystals. Hexane (about $160 \mathrm{ml}$ ) was then added to initiate the recrystallization of minoxidil. The flask was chilled in an ice bath for $4 \mathrm{~h}$ and the recrystallized minoxidil powder was collected under vacuum filtration. This material was placed in a desiccator overnight to remove residual solvent. The resultant recrystallized minoxidil had a sp. act. of about $3 \mu \mathrm{Ci} / \mathrm{mg}$.

For the first set of studies, a solution of propylene glycol, ethanol and water $(20.0: 63.2: 16.8$ $\mathrm{v} / \mathrm{v}$ ) was prepared before use. A $5 \%$ minoxidil solution was prepared by dissolving a suitable amount of the recrystallized minoxidil in this prepared vehicle. Minoxidil solutions of other concentrations were prepared by dilution of the $5 \%$ solution with plain vehicle.

For the second set of studies, a series of vehicles containing propylene glycol, ethanol and water with a fixed ratio between minoxidil and propylene glycol were prepared by substituting propylene glycol for ethanol as the concentration of minoxidil was raised. The specific compositions of these vehicles are listed in Table 1 . The minoxidil solutions of these different compositions were prepared by dissolving suitable amounts of recrystallized minoxidil in the corresponding vehicles.

\section{Permeation studies}

Abdominal skins taken from human cadavers (white females, one 80 years old and the second 66 years old) were used in the studies involving permeation of different strengths of minoxidil from a propylene glycol, water and ethanol solution of

\section{TABLE 1}

Composition of minoxidil solutions having a fixed ratio between minoxidil and propylene glycol

\begin{tabular}{lllll}
\hline $\begin{array}{l}\text { Minoxidil } \\
\text { conc. }\end{array}$ & $\begin{array}{l}\text { Propylene glycol } \\
(\% \mathrm{v} / \mathrm{v})\end{array}$ & $\begin{array}{l}\text { Ethanol } \\
(\% \mathrm{v} / \mathrm{v})\end{array}$ & $\begin{array}{l}\text { Water } \\
(\% \mathrm{v} / \mathrm{v})\end{array}$ & $\begin{array}{l}\text { Minoxidil } \\
(\mathrm{mg} / \mathrm{ml})\end{array}$ \\
\hline $1 \%$ & 10 & 70 & 20 & 10 \\
$2 \%$ & 20 & 60 & 20 & 20 \\
$3 \%$ & 30 & 50 & 20 & 30 \\
$5 \%$ & 50 & 30 & 20 & 50 \\
\hline
\end{tabular}


fixed composition. Abdominal skins of human cadavers (a 73-year-old white male and a 31-yearold white female) were used in the studies involving permeation of minoxidil from a series of solutions with fixed ratio between minoxidil and propylene glycol (Table 1). The skin samples were obtained from the University of Michigan morgue as dermatomed sections of abdominal skin, about $200 \mu \mathrm{m}$ in thickness. These were kept in a freezer at $-20^{\circ} \mathrm{C}$ and thawed before use. An appropriately sized section was cut and mounted in a Franz diffusion cell (Crown Glass, Somerville, NJ) with the stratum corneum surface facing upwards. The diffusional cross-sectional area of the skin was $0.78 \mathrm{~cm}^{2}$. The reservoir, filled with isotonic phosphate buffer ( $\mathrm{pH} 7.2)$, was equilibrated to $37^{\circ} \mathrm{C}$ before the start of the experiments. It was maintained at $37^{\circ} \mathrm{C}$ throughout each experiment by circulating water through the cell's water jacket. The receiver contents were stirred with a bar magnet.

Thirty $\mu 1$ of the formulations were added to the skin surface by a micropipet to initiate the permeation experiments. About $0.6 \mathrm{ml}$ samples were withdrawn from the receiver compartment at predetermined time intervals by a $1 \mathrm{ml}$ tuberculin syringe with a 23-gauge needle fitted with a section of PE-50 polyethylene tubing. Exactly $0.5 \mathrm{ml}$ of the samples were pipetted to $10 \mathrm{ml}$ scintillation fluid (Safety Solve, RPI, Mount Prospect, IL) and assayed on a scintillation counter. A volume of the phosphate buffer equal to that of the sample was placed back into the receiver compartment.

After correction for the dilution of sampling, the cumulative amount collected in the receiver compartment was plotted as a function of time. The flux into the receiving reservoir can be estimated from

$J \approx \frac{V}{A}\left(\frac{\mathrm{d} C}{\mathrm{~d} t}\right)$

where $J$ is the flux in $\mathrm{mg} / \mathrm{h} / \mathrm{cm}^{2}$ across the skin; $V$ is the volume of the receiver compartment (ml); $A$ is the diffusional area $\left(\mathrm{cm}^{2}\right)$ and $\mathrm{d} C / \mathrm{d} t$ is the change in concentration in terms of $\mathrm{mg} / \mathrm{ml} / \mathrm{h}$. In this study, the fluxes were estimated from the slopes of statistically drawn straight lines through all data points on the curvilinear profiles between 8 and $24 \mathrm{~h}$.

\section{Evaporation studies}

The evaporation of minoxidil solutions of different concentrations in the vehicle containing propylene glycol/water/ethanol (20.0 : 16.8 63.2) was determined by measuring weight changes of the solutions for $2 \mathrm{~h}$ at room temperature. Thirty $\mu 1$ solutions of 3 different minoxidil concentrations $\left(1 \%, 3 \%\right.$ and $5 \%$ ) were applied to a $0.78 \mathrm{~cm}^{2}$ circular area carefully mapped out on a flat surface. This was done by applying the minoxidil solutions onto a piece of masking tape, which was taped on a piece of weighing paper with a circle marked on the back. The paper together with the tape were weighed first. Each solution was weighed immediately upon its application to obtain the initial weight. The solutions were then weighed every half-hour thereafter for two hours. The weight changes were used to calculate the percentages of solution evaporated at each time interval. Crystallization was noted at the $5 \%$ concentration.

In order to more clearly observe the crystal formation and roughly establish its time dependency for different concentrations of minoxidil in this vehicle, the solutions were applied to a defined circular area blocked out on a microscope slide. About $10 \mathrm{~g}$ of paraffin (Bioloid Scientific, m.p. $50-52^{\circ} \mathrm{C}$ ) was melted in a vial and the opening of a test tube (13 $\mathrm{mm}$ in diameter) was dipped into the molten paraffin. The surface of the glass microscope slide was then touched with the top of the test tube, transferring some of the molten wax around its rim to the flat surface. The adhering molten wax formed a thick circle of wax on the slide. The area within the circle of wax was approximately the same as used in the permeation studies. Solutions of different minoxidil concentrations $(1 \%, 3 \%$ and $5 \%)$ were applied into the prescribed area and the changes in appearance of these solutions were observed at room temperature both visually (with the naked eye) and under a light microscope. After $2 \mathrm{~h}$, seeding of the $1 \%$ and $3 \%$ minoxidil solutions at room temperature was attempted by dipping a thin thread with a small amount of minoxidil powder into these solu- 


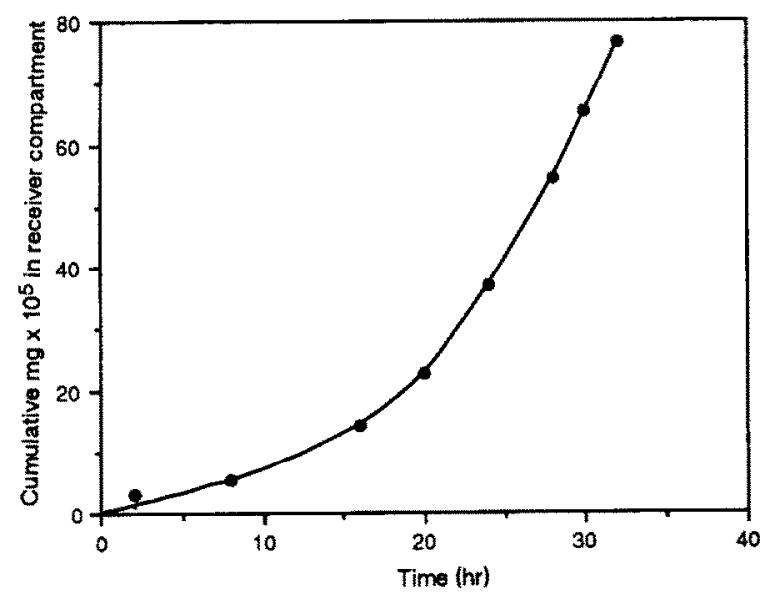

Fig. 1. Representative plot of permeation of $2 \%$ minoxidil from solutions of propylene glycol, water and ethanol through human cadaver skin.

tions and observing changes in appearance under the light microscope.

Since the rate of evaporation and the time for and degree of crystal formation of these solutions should be different at different temperatures, the changes in appearance of these solutions were observed at $35^{\circ} \mathrm{C}$, essentially the same temperature as used in the in vitro permeation studies. This was done by placing the glass slide on a stainless steel plate with a water bath beneath it.
The temperature above the glass slide was determined with a flat temperature probe.

\section{Results}

A representative permeation profile for minoxidil diffusing through a skin's section from a solution mixture of propylene glycol, water and ethanol is shown in Fig. 1. As can be seen, steady-states were not apparent in the minoxidil permeation curves. The $8-24 \mathrm{~h}$ region was used to evaluate representative flux values. Earlier time points were passed over in recognition of lag times. Time points later than $24 \mathrm{~h}$ were discarded as being impractically long (Chiang et al., 1988a). It may be noted that the minoxidil regimen typically used in hair growth is two applications per day.

The fluxes of different concentrations of minoxidil from the sample solution mixture of propylene glycol/water/ethanol (20.0:63.2:16.8 $\mathrm{v} / \mathrm{v})$ using 2 different cadaver skins are listed in Table 2. The 3-4\% minoxidil solutions yielded the highest fluxes through the $8-24 \mathrm{~h}$ time period. For each of the skins, the flux dropped back dramatically at $5 \%$ minoxidil level. Normalized fluxes for the 2 different cadaver skins, evaluated by setting the highest flux within an experiment to unity and

TABLE 2

Fluxes of minoxidil solutions of different concentrations from propylene glycol/water/ethanol vehicles (20.0:63.2:16.8, $v / v)$ using two different human cadaver skins

\begin{tabular}{|c|c|c|c|c|c|c|}
\hline & \multicolumn{6}{|c|}{ Minoxidil concentration } \\
\hline & $0.50 \%$ & $1 \%$ & $2 \%$ & $3 \%$ & $4 \%$ & $5 \%$ \\
\hline \multicolumn{7}{|c|}{ Flux of minoxidil from cadaver skin no. $1\left(\times 10^{4} \mathrm{mg} / \mathrm{cm}^{2} / \mathrm{h}\right)$} \\
\hline & 0.091 & 0.24 & 1.11 & 4.26 & 2.77 & 1.88 \\
\hline & 0.04 & 0.26 & 1.11 & 3.28 & 3.72 & 0.93 \\
\hline Mean \pm S.D. & $0.067 \pm 0.02$ & $0.22 \pm 0.05$ & $1.13 \pm 0.03$ & $3.57 \pm 0.4$ & $2.79 \pm 0.76$ & $1.55 \pm 0.55$ \\
\hline \multicolumn{7}{|c|}{ Flux of minoxidil from cadaver skin no. $2\left(\times 10^{4} \mathrm{mg} / \mathrm{cm}^{2} / \mathrm{h}\right)$} \\
\hline & 0.74 & 0.18 & 1.69 & 1.92 & 2.24 & 1.5 \\
\hline & 0.52 & 0.21 & 1.63 & 1.92 & 1.83 & 1.47 \\
\hline & 0.83 & 0.51 & 1.19 & 2.57 & 2.71 & 0.85 \\
\hline & 0.38 & 0.091 & 0.91 & 1.35 & 1.29 & 1.11 \\
\hline Mean $\pm S . D$ & $0.62 \pm 0.18$ & $0.25 \pm 0.16$ & $1.36 \pm 0.32$ & $1.94 \pm 0.43$ & $2.07 \pm 0.5$ & $1.27 \pm 0.3$ \\
\hline
\end{tabular}

Sample no. 1 and sample no. 2 are abdominal skins taken from human cadavers of an 80 -year-old white female and a 66 -year-old white female, respectively. 

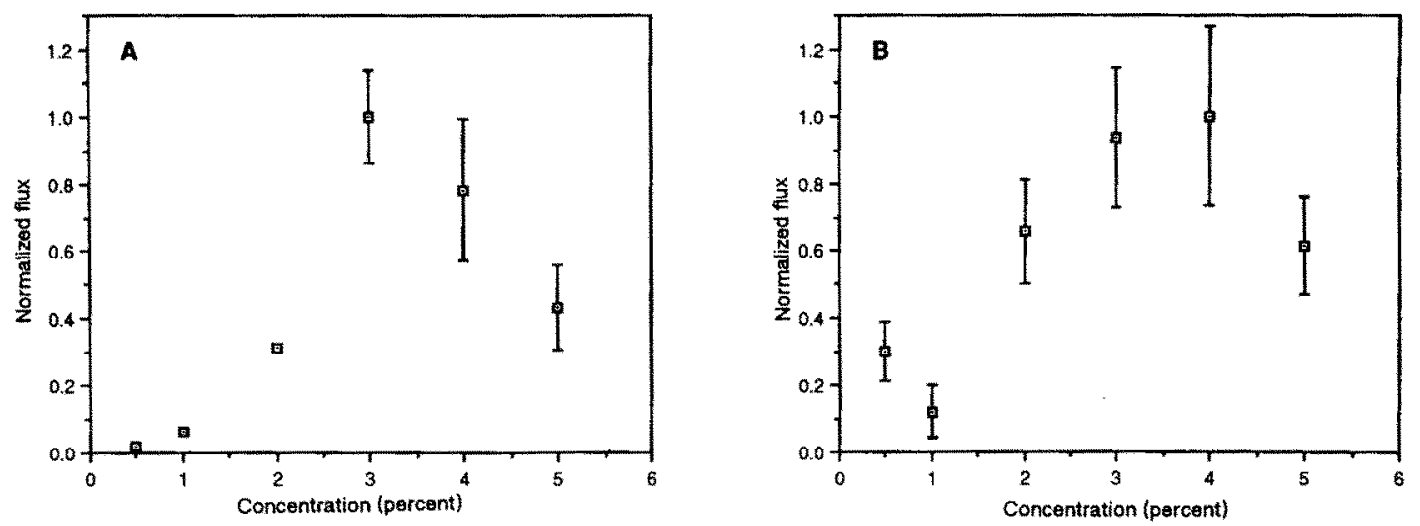

Fig. 2. Normalized plots of fluxes of minoxidil solutions of various concentrations through 2 different human cadaver skins. A: 80-year-old white female. B: 66-year-old white female.

calculating other fluxes as ratios to the maximum, are plotted in Figs. 2A and B. Table 3 summarizes the data for the permeation of different concentrations of minoxidil from a series of solutions having a fixed ratio $(1: 10)$ between minoxidil and propylene glycol.

Table 4 indicates that $65 \%$ of the weight of the minoxidil solutions evaporated within the first 30 min at room temperature. The evaporation pro-

\section{TABLE 3}

Fluxes of minoxidil solutions of different concentrations from a series of propylene glycol/water/ethanol vehicles having a fixed ratio $(1: 10)$ between minoxidil and propylene glycol using two different human cadaver skins

\begin{tabular}{|c|c|c|c|c|}
\hline & \multicolumn{4}{|c|}{ Minoxidil concentration } \\
\hline & 19 & $2 \%$ & $3 \%$ & $5 \%$ \\
\hline \multicolumn{5}{|c|}{ Flux of minoxidil from cadaver skin no. $1\left(\times 10^{5} \mathrm{mg} / \mathrm{cm}^{2} / \mathrm{h}\right)$} \\
\hline & 1.19 & 1.17 & 1.18 & 0.92 \\
\hline & 0.88 & 2.14 & 0.67 & 1.26 \\
\hline & 0.70 & 0.89 & 0.63 & 0.91 \\
\hline & 0.43 & 0.96 & 1.14 & 1.17 \\
\hline Mean \pm S.D. & $0.80 \pm 0.32$ & $1.29 \pm 0.57$ & $0.90 \pm 0.30$ & $1.07 \pm 0.18$ \\
\hline \multicolumn{5}{|c|}{ Flux of minoxidil from cadaver skin no. $2\left(\times 10^{5} \mathrm{mg} / \mathrm{cm}^{2} / \mathrm{h}\right)$} \\
\hline & 1.32 & - & - & 0.57 \\
\hline & 0.52 & - & - & 0.72 \\
\hline & 0.31 & - & - & 0.24 \\
\hline & 0.32 & - & - & 0.31 \\
\hline Mean \pm S.D. & $0.62 \pm 0.48$ & - & - & $0.46 \pm 0.22$ \\
\hline
\end{tabular}

Sample no. 1 and sample no. 2 are abdominal skins taken from human cadavers of a 73-year-old white male and a 31-year-old white female, respectively. ceeded at a much slower rate thereafter. The percentage evaporation of the different concentrations of minoxidil solutions at room temperature is summarized in Table 4. A slight influence of increasing minoxidil concentration on the net evaporation at each time point is evident in the table.

Crystallization from the $5 \%$ minoxidil solution at $35^{\circ} \mathrm{C}$ was quite evident within $10 \mathrm{~min}$ (the first crystal was observed under the microscope at about $6 \mathrm{~min}$ after application). No crystals were observed in the $1 \%$ and $3 \%$ formulations over a period of $2 \mathrm{~h}$. However, when the $3 \%$ formulation was seeded with minoxidil, copious tiny minoxidil crystals, much smaller than the ones observed in 5\% minoxidil solutions, formed immediately. Crystallization was not observed when the $1 \%$

\section{TABLE 4}

Evaporation of minoxidil solutions of different concentrations in propylene glycol/water/ethanol vehicles at room temperature.

\begin{tabular}{lllll}
\hline $\begin{array}{l}\text { Time } \\
\text { (min) }\end{array}$ & \multicolumn{2}{l}{$\begin{array}{l}\text { \% Evaporated } \\
\text { Minoxidil concentration }\end{array}$} \\
\cline { 2 - 5 } & $5 \%$ & $3 \%$ & $1 \%$ & $0 \%$ \\
\hline $0-30$ & $62 \pm 0.7^{a}$ & $63.6 \pm 0.8$ & $64 \pm 1.1$ & $65.6^{b}$ \\
$0-60$ & $65.6 \pm 0.6$ & $68.4 \pm 0.3$ & $69.2 \pm 0.2$ & $(71.8,72.9)^{c}$ \\
$0-90$ & $67.4 \pm 0.4$ & $69.6 \pm 0.4$ & $71.0 \pm 0.1$ & $(73.3,76.2)$ \\
$0-120$ & $67.8 \pm 0.9$ & $69.6 \pm 0.4$ & $71.0 \pm 0.1$ & - \\
\hline
\end{tabular}

a Mean \pm standard deviation; 3 or more experiments.

b Single experiment.

c Individual values; 2 experiments. 
minoxidil solution was seeded with minoxidil in the same fashion.

\section{Discussion}

It is known that the topical delivery of drugs from formulations is dependent on the ability of the drugs to be released from their vehicle and diffuse through the skin. As a passive diffusion process, the driving force for diffusion is the gradient of the chemical potential. In other words, the thermodynamic activity of the drug in the applied vehicle is a major factor determining the extent of a drug's partitioning into and permeation through the skin. One can argue a priori that the flux of a given drug will be proportional to the thermodynamic activity of the drug in its vehicle. One might expect that for the case where evaporation of vehicle results in concentrations beyond the drug's solubility, the flux would increase continually as volatile components of the vehicle evaporate until a saturated solution state is obtained, whereupon the drug precipitates and the activity, and consequently the flux, stabilizes. Stabilized fluxes may not occur, however, since the degree of supersaturation may be high enough to prevent precipitation. The propensity for supersaturation in a given formulation was first reported by Coldman et al. (1969) who studied the topical delivery of fluocinolone acetonide and its acetate ester formulated in vehicles with different compositions of volatile and non-volatile solvents.

At its onset, this study was planned to study concentration-flux relationships relative to topical delivery of minoxidil prepared in simple solutions of ethanol, water and propylene glycol. Previously we had shown that, by employing a simple in vitro technique, reproducible fluxes from some prototype semi-solid formulations within the same piece of skin were achievable (Chiang et al., 1988). In the present study, minoxidil solutions were applied as a thin layer to human cadaver skin in as close a facsimile to the usage situation as manageable and the fluxes of minoxidil were determined using finite dose diffusion cells (Franz cells). The $30 \mu 1$ of the minoxidil solutions that were applied to the skin resulted in an estimated application thickness of $400 \mu \mathrm{m}$. While unrealistically thick, perhaps by as much as 5- or 10-fold, this volume was chosen in order that the skin membrane might be fully overlaid with the aqueous formulations at the onset of the experiment. Otherwise, applications tended to form beads on the skin.

Representative plots for the permeation of minoxidil from solutions of the drug in a fixed propylene glycol/water/ethanol matrix do not evidence post-lag time steady-states in delivery (Fig. 1). The continuous increase in slope is almost certainly attributable to compositional changes occurring in these solutions after their application to the skin, during the permeation period. Specifically and rapidly, the vehicle's composition varies as the alcohol and water evaporate in accordance with their respective vapor pressure. Hence, the thermodynamic activity of the drug would be expected to change over the course of an experiment.

There was no precognition that a solubility problem might arise upon application of minoxidil to the skin in solutions to $5 \%$. However, the composition of the propylcne glycol/ethanol/water mixture was established based on having $20 \%$ propylene glycol in the formula and at the same time having sufficient alcohol to assure the 5\% formula would be a solution. The solubility of minoxidil in the propylene glycol/water/ethanol $(20.0: 63.2: 16.8)$ vehicle is $\approx 22 \mathrm{mg} / \mathrm{ml}$. Its solubility in ethanol and propylene glycol is $\approx 15$ $\mathrm{mg} / \mathrm{ml}$ and $90 \mathrm{mg} / \mathrm{ml}$, respectively (Upjohn, 1988). Dramatic compositional changes in the vehicle were anticipated in the test system when applied to the skin in open application and the curvilinearity of the permeation profiles came as no surprise. Since a steady-state region was not apparent for the minoxidil solution permeation curves, the $8-24 \mathrm{~h}$ region was used to generate functional flux values. Earlier time points were passed over to recognize the lag time, and later time points were discarded as being impractically long. The rationale for this choice of criteria is discussed in detail in a previous report (Chiang et al., 1988a).

The systematic increase in flux as a function of concentration was qualitatively as expected for the 
$0.5-3 \%$ minoxidil solutions (Fig. $2 \mathrm{~A}$ ) although the concave curvilinearity of the values through this region was not foreseen. The remarkably sharp fall in flux from the $3 \%$ to the $5 \%$ concentration was totally unexpected. The complete experiment, when rerun on a second piece of skin (Fig. 2B) again yielded a reduction in flux at the highest concentration tested. The unrealistically high flux from the $0.5 \%$ solution is viewed as an experimental quirk and is reported without further comment. Thus, except for the $0.5 \%$ concentration, the estimated fluxes at the various concentrations were in general agreement across the greater experiments (Table 2), and the two sets of data were pooled for statistical analysis. The fluxes seen at $0.5 \%$ and $1 \%$ minoxidil concentration are statistically lower than those at higher concentrations $(P<0.01)$. No significant difference exists between the pooled $3 \%$ and $4 \%$ results. However, the $5 \%$ result is lower than the $3 \%$ with a statistical certainty $>99 \%(P<0.01)$. After considering the latter fact, it was reasoned that crystallization might be taking place in the $5 \%$ formulation during the critical time period of the run. By implication, the $4 \%$ and perhaps the $3 \%$ solutions might be supporting supersaturation, at least for a period extending into the $8-24 \mathrm{~h}$ block of time, leading to relatively high fluxes.

To assess the possibility of supersaturation occurring in the $3 \%$ or $4 \%$ minoxidil solutions, it was desirable to know the composition changes of the minoxidil solutions during the permeation process. These were ordinarily determined from the weight changes of the minoxidil solutions over a period of $2 \mathrm{~h}$ after their application. The solution base used contains $20.0 \%$ propylene glycol $(\mathrm{v} / \mathrm{v}), 63.2 \%$ ethanol $(v / v)$ and $16.8 \%$ water $(v / v)$. These percentages correspond to $23.4 \%$ of the weight of the solution base being propylene glycol while water and ethanol constitute the other $76.6 \%$, by weight. An examination of Table 4 shows that $\approx 65 \%$ of the weight of the minoxidil solutions evaporated after the first $30 \mathrm{~min}$ at room temperature. After that, the evaporation proceeded more slowly, but by $2 \mathrm{~h}$, the weight of the solution evaporated had essentially reached a steady-state value. Considering the volatilities of the components of the mixture, this result suggests that essentially all the ethanol may have evaporated after the first halfhour and that after $2 \mathrm{~h}$, the only vehicle component remaining was propylene glycol. However, this does not establish a vehicle of fixed composition in the permeation experiments as the propylene glycol composition is also changing slowly for two reasons which we are carefully researching now. To no surprise, there is a significant transport of propylene glycol through the skin. To some surprise, the evaporative loss of propylene glycol is also significant in these experiments because it is applied as a thin film. Then, in addition to these complexities, in the permeation experiment there is a continuous flux of water up through the skin which affects the momentary composition of the vehicle.

Since ethanol is presumed to have evaporated from the vehicle very early on in the permeation process, and since ethanol is an important solvent for minoxidil, the solubility of the minoxidil in this vehicle of fixed initial solvent composition necessarily decreased markedly. Therefore minoxidil was induced to precipitate from the $5 \%$ solution. The further possibility of supersaturation at the intermediate $3 \%$ and $4 \%$ levels taking place is demonstrated through observation of the behavior of the minoxidil solutions under the microscope. For the $5 \%$ minoxidil solution, minoxidil precipitated quickly $(<10 \mathrm{~min})$. For the $3 \%$ minoxidil solution, the minoxidil remained in the vehicle for the entire $2 \mathrm{~h}$ observation period, but seeding immediately produced a copious precipitate confirming the presence of a supersaturated solution. Seeding did not induce crystallization in the $1 \%$ or the $2 \%$ minoxidil solution residues.

When the permeation of minoxidil was measured from a series of solutions of these same solvent components, but containing sufficient propylene glycol to hold the minoxidil in solution upon evaporation of the alcohol and water, no statistically significant changes in minoxidil flux were observed as the concentration of minoxidil was increased (Table 3). The result was expected since ethanol and water evaporate early in the permeation process and the resultant concentration of minoxidil in the vehicle residue is fixed. In other words, the thermodynamic activity of minoxidil in these systems was the same after a 
short equilibration period and the systems only differed in the total amount of propylene glycol covering the skin.

Based on these two sets of permeation experiments and observations on the evaporation of minoxidil solution, it appears clear that the kinetics of topical delivery of drugs are directly related to the momentary thermodynamic activity of the drug in the applied vehicle. Occasionally the drugs will supersaturate, driving them into the skin. When the concentration is pushed up to a level where prompt crystallization is unavoidable, the delivery of drugs can be markedly depressed relative to a supersaturated system even though the concentration is high. When systems are designed so as to yield an iso-thermodynamic state for different starting concentrations, the effective flux tends to remain relatively even.

\section{Acknowledgements}

This study was supported by The Upjohn Co., Kalamazoo, MI and by Grant AI/GM22303 from the National Institutes of Health.

\section{References}

Chiang, C.M., Flynn, G.L., Weiner, N.D., Addicks, W.J. and Sxpunar, G.J., Bicavailability assessment of topical delivery systems: in vitro delivery of minoxidil from prototypical semi-solid formulations. Int. J. Pharm., (1988) in press.

Chiang, C.M., Flynn, G.L., Weiner, N.D. and Szpunar, G.J., Bioavailability assessment of topical delivery systems: effect of intersubject variability on in vitro delivery of minoxidil and hydrocortisone from solution and ointment formulations. Int. J. Pharm., (1988a) in press.

Coldman, M.F., Poulsen, B.J. and Higuchi, T., Enhancement of percutaneous absorption by the use of volatile: nonvolatile systems as vehicles. J. Pharm. Sci., 58 (1969) 1098-1102.

Upjohn Company, Kalamazoo, MI, personal communication.

Weiss, V.C., West, D.P. and Mueller, C.E., Topical minoxidi in alopecia areata. J. Am. Acad. Dermatol, 5 (1981) $224-226$. 